\title{
Face Mask And Crowd Detection Using Pytorch and Multi- Task Cascade Convolutional Neural Network
}

\author{
Mukul Shende ${ }^{1}$, Amit Dravyakar², Mayur Karemore ${ }^{3}$, Lucky Khobragade ${ }^{4}$ and Davesh \\ Bondre ${ }^{5}$ \\ 1,2,3,4,5 Department of Information Technology, Priyadarshini \\ College of Engineering, Nagpur, India
}

\section{ABSTRACT}

The whole world is facing the biggest problem of health due to the rapid transmission of coronavirus disease-2019 (COVID-19). According to the World Health Organization (WHO), the best way to prevent the spread of COVID-19 is wearing a mask and keep the distance. But there is huge neglect of the guidelines by people which is resulting in daily increase in an infected patient. It is very difficult to monitor the people manually in these areas. So, in this paper, we propose an idea to monitor people using the automation process to identify the people who are wearing the mask and who are not. Many new trained models are being devised using pre-existing datasets to make the algorithm as accurate as possible. These models have made it possible to extract even the pixel details. We aim to style a binary face classifier which may detect any face present within the frame regardless of its alignment. The proposed idea or module built by pre-trained model and using computer vision libraries in python. The proposed model trained and tested on Real-world Masked Face Recognition Data set (RMFRD). By this project, we can calculate the number of people who are not wearing the mask and don't follow social distancing. By using the pre-trained libraries, this module will be robust and will have a high accuracy rate.

KEY WORDS: CONVOLUTIONAL NEURAL NETWORK, COMPUTER VISION, CORONAVIRUS, CROWD DETECTION, FACE MASK DETECTION.

\section{INTRODUCTION}

There are many types of equipment for mask detection using various libraries in python. Now to detect whether an individual is wearing a mask or not, we'll use the mask Detection Technique that uses PyTorch. Face Mask Detection Platform uses standard PC vision libraries to see if an individual wears a mask or not. The application can be related to any old or latest IP cameras to distinguish

\section{ARTICLE INFORMATION}

Received 17th Oct 2020 Accepted after revision 28th Dec 2020 Print ISSN: 0974-6455 Online ISSN: 2321-4007 CODEN: BBRCBA

Thomson Reuters ISI Web of Science Clarivate Analytics USA and Crossref Indexed Journal

\section{Clarivate
Analytics}

NAAS Journal Score 2020 (4.31)

A Society of Science and Nature Publication,

Bhopal India 2020. All rights reserved.

Online Contents Available at: http//www.bbrc.in/

Doi: http://dx.doi.org/10.21786/bbrc/13.14/82 people with/without a mask. We will see the important aspects of the model works very well not only for images having a face mask. The idea also focuses on removing the erroneous predictions which are bound to occur. Detection of face mask is performed with the help of with image processing libraries in Python. The libraries are pre-trained for the identification of masks and it gives a result with the high rate of accuracy, and this module is robust for Detection it uses ROI (Region of Interest) that means it uses the necessary points to detect the Mask. We know that there are surveillance cameras everywhere in public places. The manual detection is a very stressful job for anyone so for automating it we use this Module to Detect and it is also used to calculate the number of people. Large amounts of approaches are proposed for mask detection. The early studies on mask detection chiefly centered around utilizing customary AI algorithm to prepare successful classifiers for face mask acknowledgement and detection. Such approaches

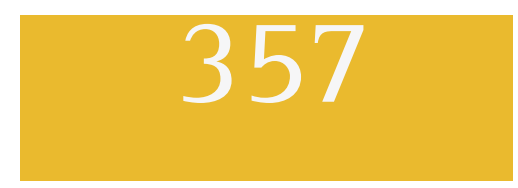


are great to some extent but the detection accuracy is relatively low.

In recent years, face detection methods based on the deep convolutional neural network have been widely studied, which are more robust and efficient than other existing methods. These strategies fundamentally actualize face identification and consequently, the area of the face bounding box, which can have a few downsides like the removed face highlights have clamour, spatial quantization is unpleasant and can't be precisely situated. These limitations will affect the following face-detection related applications. Therefore, it is necessary to study mask detection using CNN method. The module uses a Convolution algorithm which makes it more robust and accurate and advancement of this module.

As module gets input from the CCTV camera, the resolution is very low thus CNN helps to identify the face at a lower resolution. It can likewise recognize individuals without a mask by creating an alert or a warning to information security or authorities. They can see who has not covered faces with masks through software, and they can alarm them. As we use data sets to train the module to discover the face mask on the face so that data set covers a various image that includes faces with masks, faces without masks, faces with and without masks in one image and confusing images without masks and this will make this module versatile and robust.

\section{METHODOLOGY}

Our approach consists of four core principles:

A. Acquisition of image

B. Extracting features from frame and face detection

C. Determining is the person wearing a mask or not

D. Lastly calculate the crowd density in the frame

Figure 1: Mask face and crowd detection flowchart

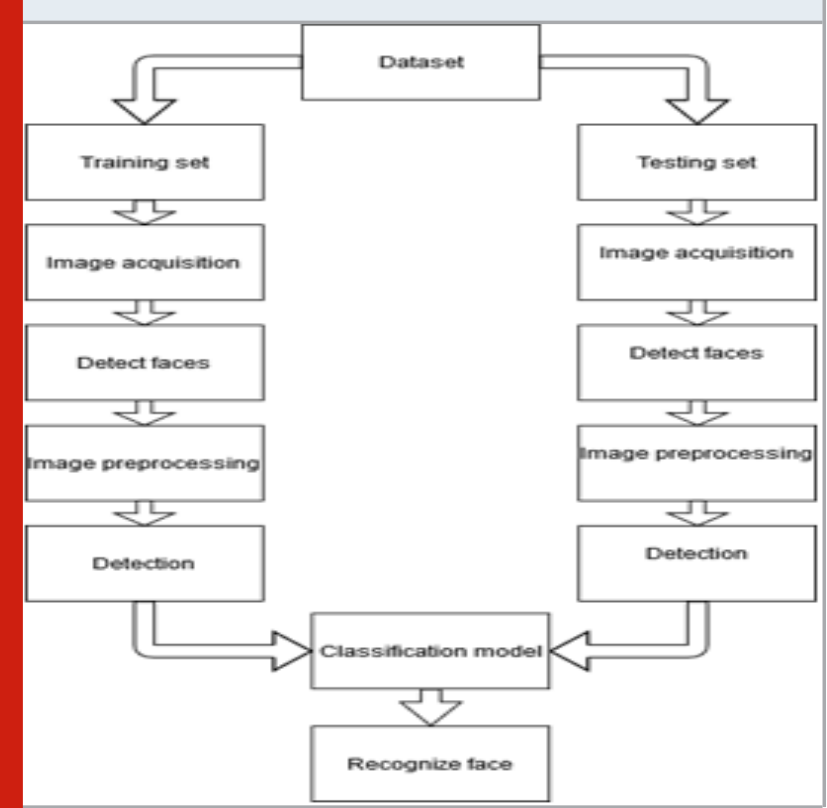

A. Image Acquisition: Image acquisition is the first generally key stage regarding the face recognition method in a subtle way. We collected masked and non-mask images from publicly available databases. These images will be used to train our module for the detection of the masked individuals and to increase the accuracy.

B. Face detection: The most essential part of our work is to detect the face in the image, without it we cannot process further. FaceNet developed on 22 deep convolutional network layers. Its output is directly trained on these deep layers to obtain a compact 128-dimensional embedding. After rectification the connected layer to be utilized as the face descriptor. These descriptors turn into a homogeneous attributepredicated descriptor utilizing the embedding module. To prepare a unique feature vector from a template, the max operator has been applied to the features. For the particular task of face apperception and verification, the network must be fine-tuned for expecting a significant boost. A very substantial amplitude of masked and nonmasked face images is acclimated to this work to re-train the FaceNet model.

Figure 2: Performance comparison between face detection packages

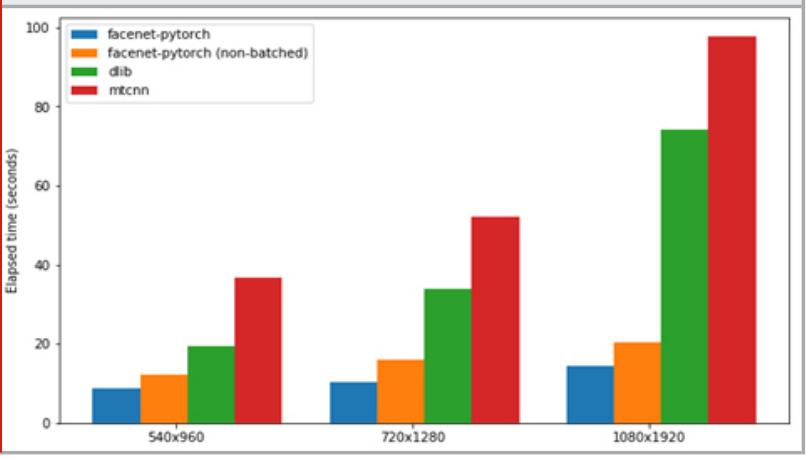

We tested with both webcam and IP camera, contrary to popular belief. Both show equivalent calculation time in a big way. This face detection for all intents and purposes is fast with 2 frames processed per second without any optimization, which definitely is quite significant. And process streams near for all intents and purposes realtime with NNPACK or generally real-time with a GPU in a generally big way.

C. Image post-processing: After face detection, crop and re-sizing methods are applied to input images. The bounding box founded in the face detection level is produced by the MTCNN model. The bounding box is then used to crop the face portion from the input images. A particular model normalized parameter is specified in the model architecture details. According to the FaceNet architecture details all the cropped images are re-sized. Few downsides like the retrieved face highlights have spatial quantization is hard and can't be precisely situated. These downsides will straightforwardly influence the subsequent ensuing face- 
related applications. Therefore, it is necessary to study mask detection using CNN method.

D. Data set construction: To build our data set, we utilized our pictures and the Region-Based Convolutional Neural Network (R-CNN). It is a group of CNN models intended for detecting object, created by Ross Girshick, there are maybe four primary varieties of the methodology, bringing about the current apex called Mask R-CNN. The Mask R-CNN is intended to figure out how to anticipate both bounding boxes for objects and for masks for those recognized. The data set we gather consists of 660 images 300 of mask and 360 on non-mask images. The age, gender, and height of persons vary for the image to image. It also comprises of different backgrounds and conditions. We partition our data-sets images into test and train set. Each train set contains $80 \%$ of all dataset as test set is roughly $20 \%$ of the complete data set. We also use publicly available data-sets and models for prediction and compare our results.

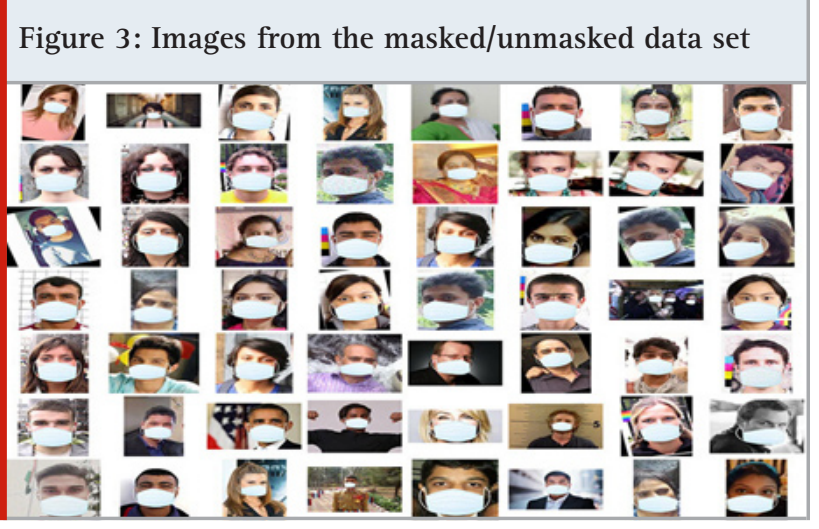

E. Crowd and mask detection: Results obtained from the MTCNN model which used to detect faces and extract features used for the detection of crowded areas where attention may be needed. The MTCNN algorithm works in three steps and uses one neural network for each in a big way. The first part particularly is a proposal network, which essentially is quite significant. It will predict potential face positions and their bounding boxes like an attention network in Faster R-CNN, which for all intents and purposes is fairly significant. The result of this step is a sort of a large number of face detection's and lots of false detection's, which for the most part is fairly significant. The second part uses images and outputs of the first prediction, or so they for all intents and purposes thought. It makes a refinement of the result to eliminate most of the false detections and aggregate bounding boxes, which is quite significant. The pretty last part refines even much more the predictions and adds facial landmarks predictions (in the basically original MTCNN implementation), Our data set model can predict if a person wearing a mask or not in a good performance range. To calculate the crowd density of a place we check if 5-6 detection boxes overlap at a single place, we can call it as crowed place and calculate the total persons present in a frame, we simply add the number of persons wearing masks and the number of persons not wearing masks.

\section{RESULTS}

For implementing real-time and correct computer vision applications on this associated system, we want to effectively optimize models throughout the model training. To train the model not solely correct but lightweight and performance efficiency in the real-time scenario. Pruning the model helps scale back the general size of the model which can lead to higher performance. This should be evaded losing accuracy as compared to the first model. All the experiments have been performed on the combination of pre-trained and added data set containing about 700 images. Out of these, $80 \%$ of images were used for training and validation while the remaining where used for testing the model. It also represents detected faces inside a highlighted rectangle concerning the prediction accuracy. Furthermore, the proposed model has also shown great leads to recognizing non-frontal faces. Alongside this, it's also ready to detect multiple facial masks during a single frame. The after processing of the model provides an outsize hike to the general accuracy. The present best accuracy of the mask and crowd counting model is $83.23 \%$.

\section{CONCLUSION}

In people that they were wearing a mask or not and if they are following social distancing in public areas. This will be contributing to public health care. By this project the workload of local authorities and police department reduces and several human errors can be corrected by this project. In this model, we used PyTorch to check the people wearing a mask or not. This proposed model also gives the result or output by processing the images and real-time videos streaming (CCTV camera). We can say how accurately our model work. Besides, the system is straightforward to deploy into any existing system of a business while keeping the security and privacy of users' data. Thus, the mask detection framework goes to be the advanced answer for some enterprises, particularly retail, medical services, and public areas. After this pandemic, this technology is used for detecting accidents on road and for crowd management in the public places. This document we have proposed a novel face mask detector, this will help to cut the fatigue of local authorities to check manually.

\section{REFERENCES}

G. Deore, R. Bodhula, V. Udpikar and V. More, "Study of masked face detection approach in video analytics," 2016 Conference on Advances in Signal Processing (CASP), Pune, 2016, pp. 196-200, doi: 10.1109/ CASP.2016.7746164.

J. Wang and Z. Xu, "Crowd anomaly detection for automated video surveillance," 6th International Conference on Imaging for Crime Prevention and Detection (ICDP-15), London, 2015, pp. 1-6, doi: 
10.1049/ic.2015.0102.8274819.

Meenpal, Toshan \&t Balakrishnan, Ashutosh \&t Verma, Amit. (2019). Facial Mask Detection using Semantic Segmentation. 1-5. 10.1109/CCCS.2019.8888092.

Saqib, Muhammad \&t Khan, Sultan \&t Sharma, Nabin Et Blumenstein, Michael. (2019). Crowd Counting in Low-Resolution Crowded Scenes Using Region-Based Deep Convolutional Neural Networks. IEEE Access. PP.
1-1. 10.1109/ACCESS.2019.2904712.

W. Bu, J. Xiao, C. Zhou, M. Yang and C. Peng, "A cascade framework for masked face detection," 2017 IEEE International Conference on Cybernetics and Intelligent Systems (CIS) and IEEE Conference on Robotics, Automation and Mechatronics (RAM), Ningbo, 2017, pp. 458-462, doi: 10.1109/ICCIS.2017 